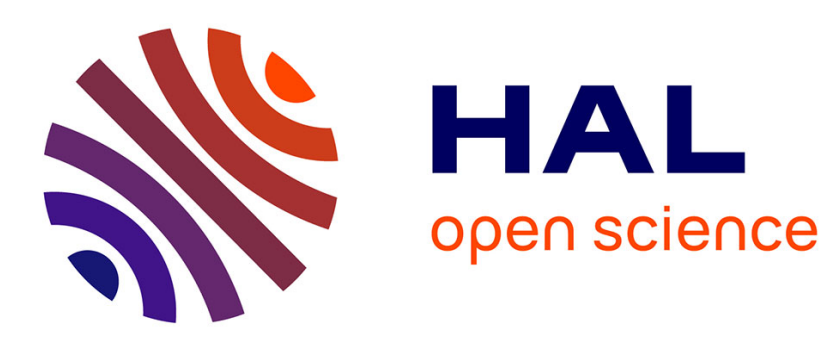

\title{
Core level photoemission from (111)-type InAs surfaces
}

\author{
C. Andersson, L. Olsson, M. Håkansson, L. Ilver, U. Karlsson, J. Kanski
}

\section{To cite this version:}

C. Andersson, L. Olsson, M. Håkansson, L. Ilver, U. Karlsson, et al.. Core level photoemission from (111)-type InAs surfaces. Journal de Physique IV Proceedings, 1994, 04 (C9), pp.C9-209-C9-212. 10.1051/jp4:1994937 . jpa-00253498

\section{HAL Id: jpa-00253498 https://hal.science/jpa-00253498}

Submitted on 1 Jan 1994

HAL is a multi-disciplinary open access archive for the deposit and dissemination of scientific research documents, whether they are published or not. The documents may come from teaching and research institutions in France or abroad, or from public or private research centers.
L'archive ouverte pluridisciplinaire HAL, est destinée au dépôt et à la diffusion de documents scientifiques de niveau recherche, publiés ou non, émanant des établissements d'enseignement et de recherche français ou étrangers, des laboratoires publics ou privés. 


\title{
Core level photoemission from (111)-type InAs surfaces
}

\author{
C.B.M. Andersson, L.Ö. Olsson*, M.C. Håkansson**, L. Ilver*, U.O. Karlsson and J. Kanski* \\ Department of Physics, Royal Institute of Technology, 10044 Stockholm, Sweden \\ * Department of Physics, Chalmers University of Technology, 43040 Göteborg, Sweden \\ ** Department of Synchrotron Radiation Research, University of Lund, 22362 Lund, Sweden
}

\begin{abstract}
The InAs(111)2x2 and InAs( $\overline{1} \overline{1} 1) 1 \times 1$ surfaces have been studied with high resolution core level spectroscopy. For the InAs(1) $1 \overline{1}) 1 \times 1$ surface both the In $4 \mathrm{~d}$ and the As $3 \mathrm{~d}$ core levels display strong surface core level shifts, while for the InAs(111)2x2 surface only the In $4 \mathrm{~d}$ level shows a detectable surface shift. The results indicate that the InAs( $1 \overline{1} \overline{1}) 1 \times 1$ surface is relaxed, with atom layer displacement extending to subsurface layers. Unexpectedly, we find no surface shifted anion core level for the InAs(111) $2 \times 2$ surface.
\end{abstract}

\section{INTRODUCTION}

The atomic and electronic structure of the polar (111) surfaces of III-V semiconductors have gained an increased attention during recent years [1-8]. Depending on crystal orientation and stoichiometry, the (111)type surfaces display different atomic geometries. The most common reconstruction for the clean (111) surface is $2 \times 2$, as seen in low energy electron diffraction (LEED), while the (i11 $)$ surface displays several reconstructions as function of surface composition.

In this paper we report results from high resolution core level spectroscopy studies of the InAs(111)2x2 and $\operatorname{InAs}(\overline{1} \overline{1}) 1 \times 1$ surfaces. Since different geometrical configurations are reflected via different binding energies, core level spectra can be utilized to provide direct information about the presence of inequivalent surface sites and their relative abundance. In this way core level spectroscopy serves as an important complementary tool for structural determinations. Increased knowledge about the surface geometries for the clean surfaces may be significant for utilizing InAs in high-speed electronic-device applications, since the formation of high quality heteroepitaxial interfaces are of supreme importance in such devices.

\section{EXPERIMENTAL}

The experiments were performed at the toroidal-grating monochromator beam line at the MAX synchrotron radiation facility in Lund, Sweden [9]. The angle resolved photoelectron spectra were recorded 
with a modified VSW ARIES system. Interlocked with the photoemission system is a molecular beam system equipped with six effusion cells and RHEED.

The InAs substrates were sulphur doped and were supplied by MCP Wafer Technology Limited, England. The InAs(111) $1 \times 1$ and the InAs(111)2×2 surfaces were prepared by repeated cycles of sputtering and annealing. This preparation procedure yielded well ordered surfaces, which showed sharp LEED patterns with a low background. Both surfaces could also be grown by MBE, and showed the same photoemission spectra.

\section{RESULTS AND DISCUSSION}

Figure 1a shows In $4 \mathrm{~d}$ and As $3 \mathrm{~d}$ spectra from the $\operatorname{InAs}(111) 2 \times 2$ surface measured at $0^{\circ}$ and $60^{\circ}$ emission angles, i.e. at different surface sensitivities. Also shown in the figurare decompositions into spin-orbit split Voigt functions. The fitting parameters are given in Table 1. It is seen from the figure that the In $4 \mathrm{~d}$ spectra can be constructed from two different components, while only one component is necessary for the As $3 \mathrm{~d}$ core level. From the intensity variations between the two In $4 \mathrm{~d}$ components as function of emission angle and photon energy it is clear that the component towards higher binding energy is surface related.

In figure $1 \mathrm{~b}$ the In $4 \mathrm{~d}$ and $\mathrm{As} 3 \mathrm{~d}$ core levels from the $\operatorname{InAs}(\overline{1} \overline{1}) 1 \mathrm{x} 1$ surface are displayed together with the corresponding decompositions. From the raw data it is clearly seen that both the In $4 \mathrm{~d}$ and the As $3 \mathrm{~d}$ spectra are broader than the corresponding spectra from the $\operatorname{InAs}(111) 2 \times 2$ surface. A deconvolution based on similar parameters as used for the $(2 \times 2)$ surface shows that at least two components are needed to adequatly fit the experimental data. For the As $3 \mathrm{~d}$ level we note that both components have about the same intensity independent of emission angle. The identification of the As $3 \mathrm{~d}$ bulk component was accomplished by increasing the photon energy and thereby the bulk sensitivity. Additionally, we have used the established separation between the In $4 \mathrm{~d}$ and As $3 \mathrm{~d}$ bulk components. The lack of an intensity enhancement of the surface component as function of emission angle may be due to diffraction effects, which are known to be strong in this energy range from III-V semiconductors [11]. These effects could in principle be used for a geometrical structure determination. This would, however, require substantially more data on the angle- and energy dependence.
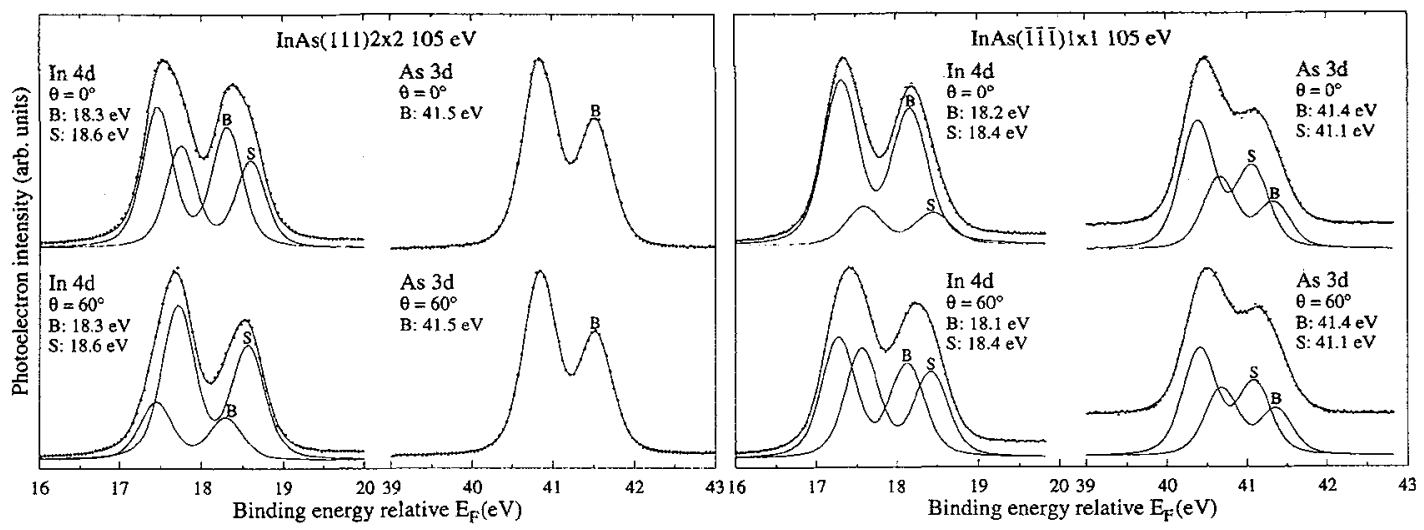

Figure1 Core level spectra from the InAs(111)2x2 and InAs( $\overline{111}) 1 \times 1$ surfaces. In each case the dots represent the experimental data, while the lines show the fitted curves. 
The (111) surfaces of III-V semiconductors have been subject to extensive experimental and theoretical studies. Based on LEED investigations Tong et al. proposed a vacancy-buckling model for the $\mathrm{GaAs}(111)$ surface with one of four surface gallium atoms missing [1]. Robinson et al. studied the $\mathrm{InSb}(111) 2 \times 2$ and $\mathrm{GaSb}(111) 2 \times 2$ surfaces by X-ray diffraction and found that the vacancy-buckling model could well explain the experimental data if the distortions were permitted to extend down to the second layer [4]. The vacancy model for the (111) $2 \times 2$ surfaces was given further support when Haberern and Pashley could resolve individual surface gallium atoms in their scanning tunneling microscopy images of the $\mathrm{GaAs}(111) 2 \times 2$ surface [5].

\begin{tabular}{|c|c|c|c|c|c|c|c|c|}
\hline & \multicolumn{4}{|c|}{$\operatorname{InAs}(111) 2 \times 2$} & \multicolumn{4}{|c|}{$\operatorname{InAs}(\overline{111}) 1 \mathrm{x} 1$} \\
\hline & \multicolumn{2}{|c|}{ In $4 d$} & \multicolumn{2}{|c|}{ As $3 d$} & \multicolumn{2}{|c|}{ In $4 d$} & \multicolumn{2}{|c|}{ As 3d } \\
\hline & $0^{\circ}$ & $60^{\circ}$ & $0^{\circ}$ & $60^{\circ}$ & $0^{\circ}$ & $60^{\circ}$ & $0^{\circ}$ & $60^{\circ}$ \\
\hline s-o splitting & 0.855 & 0.855 & 0.685 & 0.685 & 0.855 & 0.855 & 0.685 & 0.685 \\
\hline branching ratio & 1.18 & 1.37 & 1.52 & 1.54 & 1.23 & 1.29 & 1.58 & 1.47 \\
\hline surface shift & -0.27 & -0.29 & - & - & -0.29 & -0.29 & 0.27 & 0.27 \\
\hline $\begin{array}{l}\text { surface/bulk } \\
\text { intensity ratio }\end{array}$ & 0.72 & 2.69 & - & - & 0.23 & 0.91 & 1.77 & 1.58 \\
\hline$\Delta \mathrm{E}_{\text {Gaussian }}$ & 0.33 & 0.38 & 0.40 & 0.40 & 0.41 & 0.40 & 0.40 & 0.40 \\
\hline$\Delta \mathrm{E}_{\text {Lorentzian }}$ & 0.175 & 0.175 & 0.16 & 0.16 & 0.175 & 0.175 & 0.16 & 0.16 \\
\hline
\end{tabular}

Table 1 Fitting parameters for core level spectra from $\operatorname{InAs}(111) 2 \times 2$ and $\operatorname{InAs}(\overline{111}) 1 \times 1$ surfaces. The Lorentzian was kept constant in the fitting process.

Given the similarities in the atomic and electronic structure for the other III-V surfaces it is likely that the vacancy model should apply also for the InAs(111)2x2 surface. This is partly in agreement with our core level spectra. In the vacancy-buckling model for the $2 \times 2$ reconstruction the surface group III and group V atoms are rearranged in such a way that the surface geometry locally is reminiscent of the non-polar (110) surface. Disregarding possible differences in the final state one would therefore expect the surface corelevel shifts to be similar to the shifts measured for the (110) surface. For the In $4 \mathrm{~d}$ core level this is indeed the case. The present results show a surface core level shift of $0.28 \mathrm{eV}$, which is similar to the $0.27 \mathrm{eV}$ found for the (110) surface [10]. In the case of As 3d, however, the situation is quite different. On the (110) surface the As $3 \mathrm{~d}$ level displays a surface shift of $0.30 \mathrm{eV}$ [11], but on the (111)2x2 surface we can not resolve any shifted component - the As $3 \mathrm{~d}$ line shape is independent of emission angle and the spectrum is well fitted with a single component. A similar result is obtained on the corresponding InSb surface[12]. Considering, in addition, that the vacancy-buckling model includes two types of surface anion sites, namely the three anions which surround a vacant cation site and a single anion, not adjacent to any vacancy site, the absence of any significant shifted As 3d component in the present spectra is surprising. Our observation may possibly be understood on the basis of charge density calculations by Kaxiras et al. [3]. Within the vacancy-buckling model these authors found that the three As atoms around the vacancy site relax toward this site and attain an almost $\mathrm{p}^{3}$ bonding configuration. Furthermore, the single As atom which is not adjacent to any vacancy site could also be assumed to attain a $\mathrm{p}^{3}$ bonding configuration. It could then be argued that the local charge arrangement at the surface anions is rather similar to that in the bulk, and consequently, the surface core level shifts would be very small. It is then also assumed that all other effects which contribute to 
surface shifts (Madelung potential, final state screening) are either similar to the corresponding bulk properties, or tend to cancel eachother. Clearly, further studies of this phenomenon are of interest.

Several III-V (111) surfaces display a 1x1 LEED pattern [6-8]. Hou et al. studied the $\operatorname{InP}(\overline{1} 1 \overline{1}) 1 \times 1$ surface by angle resolved photoelectron spectroscopy and compared the experimentally obtained dangling bond dispersion with a theoretical calculation based upon a slab model and the extended Hückel theory [6].The results showed that the InP( $\overline{11} \overline{1}) 1 \times 1$ surface is a relaxed surface with the atomic layer displacement extended to the outermost four layers. The dangling bond dispersion found by Hou et al. for the $\operatorname{InP}(\overline{1} \overline{1}) 1 \times 1$ surface is very similar to dangling bond dispersion for the $\operatorname{InAs}(\overline{1} \overline{1} \overline{1}) 1 \times 1$ surface [8]. This indicates that also this surface exhibits a displacement of the subsurface atoms. It is therefore likely that both the In and the As should show surface shifted components, in good agreement with our experimental observations.

\section{SUMMARY}

Surface sensitive high resolution core level spectroscopy has been applied to the InAs(111)2x2 and $\operatorname{InAs}(\overline{\mathbf{1}} \overline{1} \overline{1}) 1 \times 1$ surfaces. For the InAs $(\overline{1} \overline{1} 1) 1 \times 1$ surface both the In $4 \mathrm{~d}$ and the As $3 \mathrm{~d}$ core levels displayed strong surface core level shifts, while for the InAs(111)2x2 surface only the In $4 \mathrm{~d}$ level showed a detectable surface shift. The results indicates that the $\operatorname{InAs}(\overline{11} \overline{1}) 1 \times 1$ surface is a relaxed surface with atom layer displacement extending to subsurface layers. For InAs(111)2x2 no surface shifted As core level is detected, which may possibly be due to similar local charge density on the surface anions as in the bulk.

\section{ACKNOWLEDGEMENTS}

We would like to thank the MAX-lab staff for their most generous assistance. This work has been financially supported by the Swedish Natural Science Research Council.

\section{REFERENCES}

1. Tong S.Y., Xu G., and Mei W.N., Phys Rev. Lett. 52 (1984) 1693.

2. Chadi D.J., Phys. Rev. Lett. 52 (1984) 1911.

3. Kaxiras E., Bar-Yam Y., Joannopoulos J.D., and Pandey K.C., Phys. Rev. B 35 (1987) 9625.

4. Robinson I.K., Bohr J., Feidenhans'1 R., Nielsen M., Grey F., and Johnson R.L., Surf. Sci. 217 (1989) L435.

5. Haberern K.W. and Pashley M.D., Phys. Rev. B 41 (1990) 3226.

6. Xiao-yuan Hou, Gou-sheng Dong, Xun-min Ding, and Xun Wang, Surf. Sci. 183 (1987) 123.

7. Daoxuan Dai, Xiangdong Wang, Jihuang Hu, and Yuqing Ge, Surf. Sci. 294 (1993) 349

8. Andersson C.B.M., Karlsson U.O., Håkansson M.C., Olsson L.Ö., Hver L., Kanski J., Nilsson P.O., and Persson P.E.S., Surf. Sci. in press.

9. Karlsson U.O., Andersen J.N., Hansen K., and Nyholm R., Nucl. Instrum. Methods Phys. Res. A 282, (1989) 553.

10.Andersen J.N. and Karlsson U.O., Phys. Rev. B 41 (1990) 3844

11.Gota S., Gunnella R., Wu Zi-Yu, Jézéquel G., Natoli C.R., Sébilleau D., Bullock E.L., Proix F., Guillot C., and Quémerais A., Phys. Rev. Lett. 71 (1993) 3387.

12.Andersson C.B.M., Karlsson U.O., Håkansson M.C., Olsson L.Ö., Ilver L., and Kanski J., to be published 